\title{
Sistema de Información Agrícola para la disminución de Brechas entre Oferta y Demanda - AGROCRAFT
}

\author{
Urbano E. Gómez, Jesika P. Pérez y José L. Ramírez \\ Facultad de Ingeniería de Sistemas e Informática, Universidad Pontificia Bolivariana (UPB), Autopista a \\ Piedecuesta Km 7, Edificio I, Oficina 301, Bucaramanga, Colombia (e-mail: urbano.gomez@upb.edu.co; \\ jesika.perez19@gmail.com; sid.josep@gmail.com)
}

Recibido Nov. 10, 2015; Aceptado Ene. 15, 2016; Versión final Feb. 19, 2015, Publicado Jun. 2016

\begin{abstract}
Resumen
Este artículo presenta un sistema de información soportado en tecnologías web y móvil para gestionar la comercialización de productos agrícolas entre organizaciones consumidoras y productoras asociadas y mitigar la brecha de la oferta y la demanda. Los productores asignan, desde un dispositivo móvil, la producción de sus cultivos según las necesidades de las organizaciones consumidoras. El software hace una estimación de la fecha y la cantidad de producción de sus futuras cosechas. Esto se hace mediante un modelo que opera en base a información sobre el área a sembrar y el producto escogido. Las organizaciones consumidoras gestionan, desde un navegador web, sus necesidades productivas a partir de los cultivos registrados por las productoras y las cosechas realmente obtenidas.
\end{abstract}

Palabras clave: sistema de información; producción agrícola; toma de decisiones; oferta y demanda

\section{Agricultural Information System for the reduction of the gap between Supply and Demand - AGROCRAFT}

\begin{abstract}
This article presents an information system supported by mobile and web technologies to manage the marketing of agricultural products between consumer organizations and producers groups and mitigate the gaps of supply and demand. Producers assign, from a mobile device, the crop production according to the needs of the consumer organizations. The software estimates the timing and amount of future production of their crops. This is done through a model that operates based on information about the cultivated area and the chosen product. The consumer organizations manage from a web browser, their needs based on registered producers and the crop really obtained.
\end{abstract}

Keywords: information system; agricultural production; decision making; supply and demand 


\section{INTRODUCCIÓN}

La presencia de las tecnologías de la información y la comunicación para automatizar y optimizar procesos agrícolas, aumentar la rentabilidad y productividad de los recursos económicos e incluir la asistencia técnica, permite ofrecer productos y servicios más adecuados a las necesidades y ampliar las oportunidades de progreso en el sector (Cárdenas, 2012). AGROCRAFT tiene como propósito equilibrar los niveles de oferta y demanda permitiendo generar estrategias que mejoren el sector agropecuario y contrarresten el hecho de que en este sector, los productos que generan los agricultores para la comercialización (oferta) superan en gran medida o son insuficientes con respecto a la cantidad de productos que los consumidores requieren (demandan), lo que ocasiona fluctuaciones en los precios de los mismos.

AGROCRAFT integra las organizaciones productoras y transformadoras de productos agrícolas y tiene en cuenta las actividades, eventos, recursos y como se deben coordinar para su integración con las funciones que gestionan y controlan los procesos de siembra, cosecha y acopio. En este trabajo se presenta un software que estima la producción agrícola a partir de un modelo definido para cada producto, es decir, realiza el cálculo de la cantidad en kilogramos que probablemente el agricultor obtendrá en una cosecha para determinada fecha y relaciona esta producción estimada con las necesidades productivas del consumidor. Esto permite conocer de manera oportuna el posible nivel de satisfacción a previas necesidades registradas por el consumidor. Además, se genera información para la toma de decisiones y se disminuye la incertidumbre de a quien vender y a quien comprar, al integrar las dos partes interesadas en el mismo sistema.

\section{ANTECEDENTES}

Las TIC han cambiado la forma de vida de los seres humanos, en el sector agrícola la transformación de la producción y comercialización, se debe en gran parte a la incorporación de las TIC en los procesos, para mejorar la competitividad y optimizar la producción por medio del acceso oportuno a la información. Por ello, en Colombia se han desarrollado para la agroindustria algunos aplicativos que operan sobre dispositivos móviles, dos de ellos son: Agroclima y Agropecios (Agronet, 2015): i) Agroclima es una aplicación que toma los datos climáticos como lluvias y temperatura para generar gráficas de tendencias que permiten identificar el momento oportuno para la siembra de productos agrícolas y orientar al productor en las cantidades de agua que necesita el cultivo; y ii) Agropecios presenta los comportamientos de precio de los productos agrícolas de manera diaria, semanal y mensual, permitiendo de igual manera comparar los precios de un Producto en los diferentes mercados que le puedan interesan al usuario

A nivel mundial son presentados cinco ejemplos de proyectos similares a AGROCRAFT, estos son:

i) Lee (2013) presenta un sistema de información que propone equilibrar la oferta y demanda de los productos agrícolas haciendo uso del internet de las cosas mediante un análisis de correlación entre la información estadística que se registra de los cultivos y la información del entorno agrícola identificada por sensores.

ii) Mubin (2012) presenta una aplicación cliente/servidor para facilitar a los agricultores el acceso a información contextual relevante para seleccionar las plantas que no están creciendo o produciendo adecuadamente y lograr procesos más productivos con menor esfuerzo y poder revisar los aspectos que llevaron a ello, obteniendo una plataforma para la investigación agrícola.

iii) Erden (2015) describe un monitoreo del crecimiento de la agricultura en Turquía a través de sistemas de información geográfica y sensores remotos con el fin de cultivar en sus hábitats con la máxima eficiencia, para mantener el equilibrio de la oferta y la demanda, y buscar el desarrollo sostenible.

iv) La OECD - La Organización para la Cooperación y el Desarrollo Económicos - (2012), coordina políticas económicas y sociales cuenta con un proyecto para la evaluación de la evolución de la política agrícola y de apoyo y el fomento de la innovación y el crecimiento de la productividad en la agricultura en la Unión Europea, Canadá, Australia y Chile y para ello cuenta con un sistema de Información web de Cultivos (SICI) que permite además de la gestión de estos dos propósitos, el control sobre ensayos de cultivos brindando ayuda en la toma de decisiones al facilitar el pronóstico de la producción.

v) Earth Innovation Institute - Instituto de Innovación Tierra - (2015), está desarrollando condiciones de gobernabilidad regional mediante el desarrollo rural inclusivo es soportado en un Sistema de seguimiento en línea y en el que sean sostenibles las cadenas de suministro de productos agrícolas y en la que se dé una transición para un equitativo y sostenible suministro de productos básicos y el desarrollo rural. Entre algunas ventajas que han obtenido es el aprovechamiento de los costos de oportunidad, disminución de la deforestación y una vinculación constante de los pequeños productores. 


\section{PROPUESTA METODOLÓGICA}

La metodología utilizada tomó como base el desarrollo ágil SCRUM, el cual brinda un conjunto de buenas prácticas basada en el trabajo colaborativo para entregar mejores resultados en el desarrollo de un proyecto por medio de la segmentación de tareas y asignación de roles (Alaimo, 2015). El proceso de trabajo comienza con la elaboración de la lista de historias de usuario (Product Backlog) propias de un requerimiento funcional, las cuales tiene un tiempo de ejecución de 1 a 2 semanas (Sprints), segmentadas por actividades, cada una con duración máxima de desarrollo de 8 horas para finalmente ser analizadas, verificadas y aprobadas en un lapso de 2 a 3 días y aumentar el valor del producto que se desea entregar, como se describe en la figura 1.

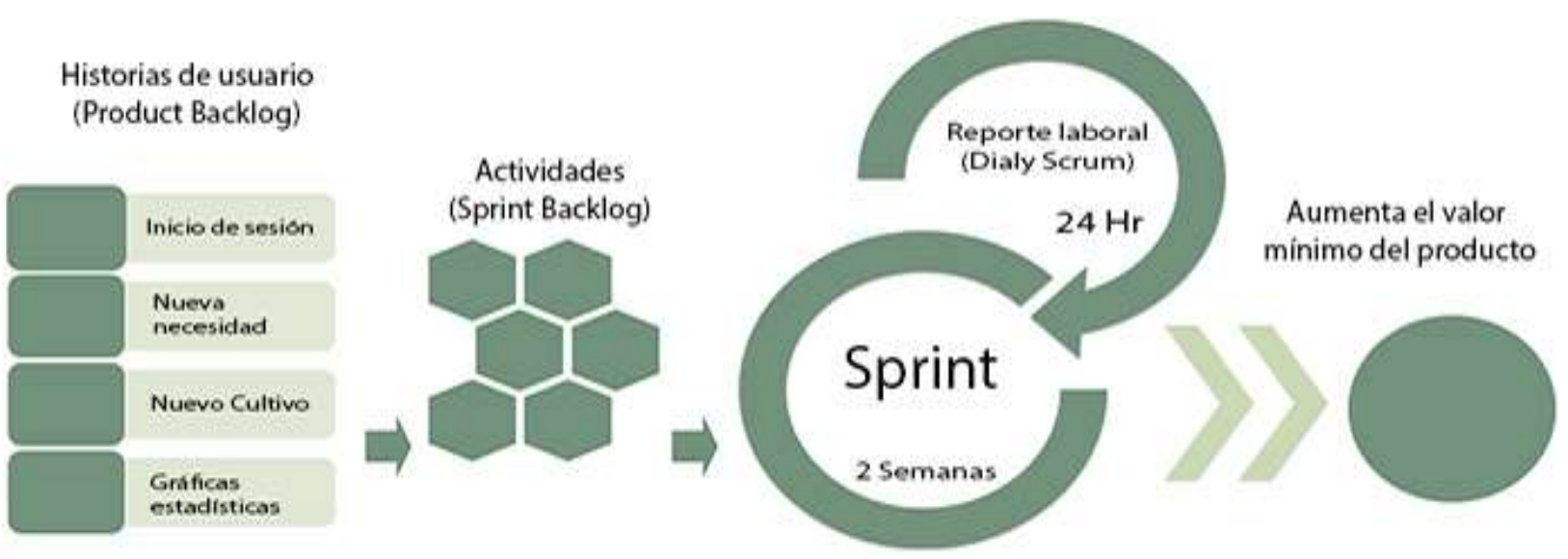

Fig. 1. Metodología del Proyecto (Adaptada de Alaimo, 2015)

Adicionalmente, el desarrollo se apoyó en el sistema Subversion en el control de versiones (CVS) para gestionar los cambios realizados y permitir la recuperación a otra versión (Subversion, 2004).

\section{RESULTADOS}

En el proceso para comenzar a equilibrar la oferta y demanda de los productos agrícolas se logró producir un sistema de información robusto con la aplicación de tecnologías y herramientas innovadoras que permite una correcta gestión de los procesos de abastecimiento y las de estrategias que mejoren el sector agropecuario.

\section{Roles de usuario}

El sistema se encuentra segmentado por diferentes tipos de usuarios que pueden acceder a AGROCRAFT, como se observa en la Fig. 2, quienes a través de sus respectivos roles permiten el cumplimiento satisfactorio de su propósito, los cuales se especifican a continuación y se representan en la Fig. 3, en donde es presentado un diagrama general de secuencia en el que aprecian las principales acciones de cada uno de los roles).

Administrador: se encarga de parametrizar la herramienta, es decir, gestionar los usuarios vinculados y los productos con sus modelos, los cuales son utilizados por el software para el cálculo de la cantidad estimada de producción de un cultivo, teniendo en cuenta que los modelos representan la producción a obtener en kilogramos por metro cuadrado o hectárea que se cultive de un determinado producto, seguido por tiempo que tardará en cosecharse. El Sistema permite al administrador acceder a los reportes estadísticos que muestran cómo se están satisfaciendo las necesidades de las organizaciones y la diferencia que exista entre la producción calculado a través de un modelo y lo que realmente se está produciendo en la cosecha.

Organización: se encargan de gestionar las necesidades productivas de sus sucursales de abastecimiento, las cuales hacen referencia a la cantidad de producto que se requiere para las fechas de corte en un día determinado de la semana, en los cuales se realiza el proceso de abastecimiento. Cuando cada agricultor ingresa las cosechas (y la organización va recibiendo los productos) puede generar la información para conocer las necesidades no suplidas por los mismos.

Agricultor (Productora): se encarga de satisfacer las necesidades productivas de las organizaciones a través de sus cultivos y proporcionar las cantidades finales producidas de sus cosechas. 


\section{Modelo de Producción Estimada}

El modelo para el cálculo de la producción estimada de un cultivo, esta soportado en la base de datos, particularmente en las tablas Producto y Modelo y en una ecuación. En la tabla Modelo, se almacenan las cantidades promedio que se producen de un producto en cada unidad de superficie y el tiempo de producción estimado en semanas, para que cuando un agricultor desee registrar un cultivo y asignarlo a la necesidad productiva de una organización, la ecuación, calcule la cantidad que se obtendría y establezca la fecha posible de la cosecha.

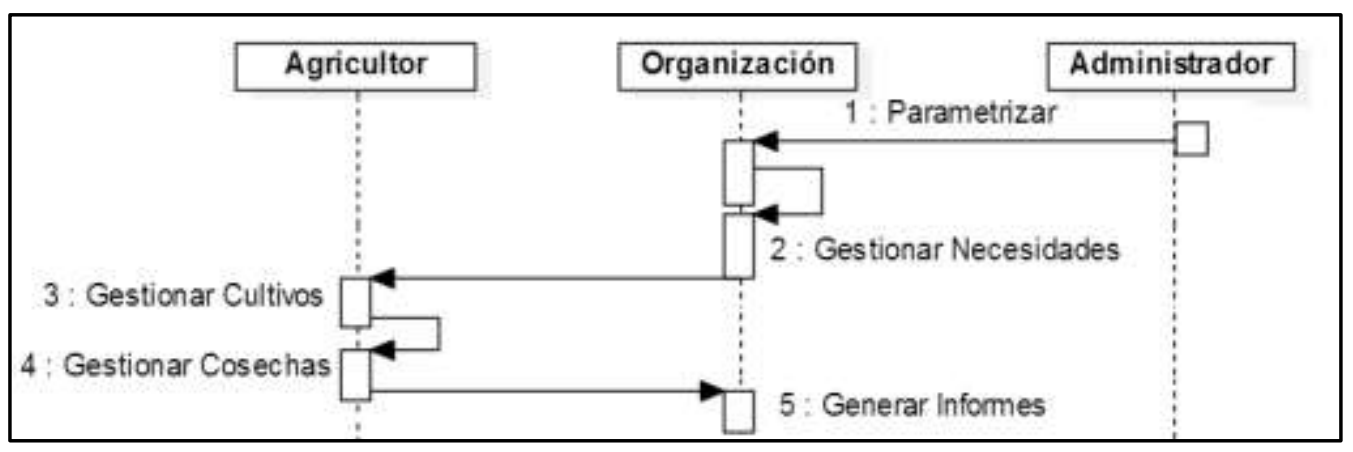

Fig. 2. Representación de las acciones para cada uno de los roles o perfiles de usuario

Para el registro de un cultivo, el agricultor debe seleccionar el producto y la cantidad de superficie a sembrar en metros cuadrados o hectáreas y la herramienta calcular la producción y fecha estimada, para proporcionar una lista de necesidades productivas de las organizaciones registras que requieren el producto en menor o igual cantidad en la fecha estimada, para que el agricultor seleccione una opción y registre el cultivo. Un ejemplo es presentado en la Fig. 3:. Un producto de ejemplo registrado es el maíz, en donde una hectárea produce 1.573 kilogramos y un metro cuadrado produce 0.16 kilogramos de maíz tradicional en un lapso de 80 a 200 días (Grande et al., 2012)
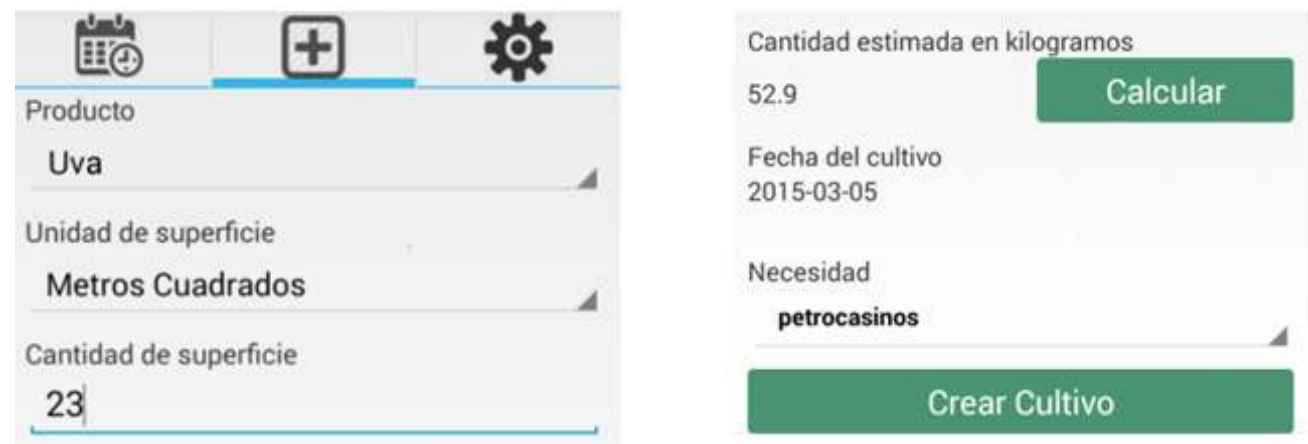

Fig. 3: Registro de un Cultivo (tomada del dispositivo móvil)

Informe para el Análisis del grado de satisfacción de las necesidades productivas

Las organizaciones pueden identificar, el nivel de satisfacción de cada una de sus necesidades productivas consolidada de la organización o por sucursal, en la Fig. 3 se puede observar el alcance propuesto de la oferta que se tendrá para los siguientes seis meses con respecto a la demanda propuesta por el administrador.

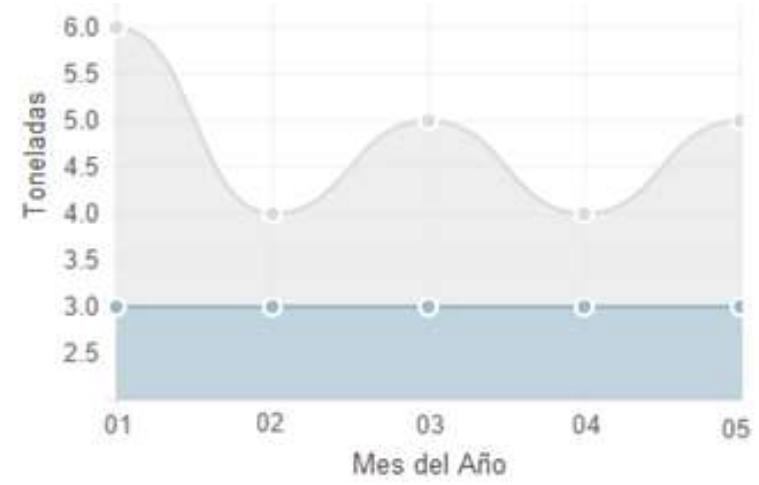

Fig. 3. Gestión de Necesidades Productivas (tomada desde la página web). 


\section{Arquitectura del Sistema de Información}

En la Fig. 4 se observa la arquitectura distribuida que utiliza el sistema de información, la cual presenta una serie de servicios proporcionados por uno o varios servidores a un conjunto de clientes que consumen estos servicios, mitigando problemas de escalabilidad y rendimiento para optimizar la transferencia de información entre el servidor web y el servidor de la base de datos.

\section{Aspectos de Seguridad}

La importancia de la información que se maneja en los procesos exigen la protección de ataques y la mitigación de sus vulnerabilidades, por ello AGROCRAFT cuenta con:

i) Cifrado de datos para el almacenamiento de la contraseña de usuario, proceso que se efectúa a través de una función hash, la cual realiza una operación matemática sobre el conjunto de datos para transformarlo en una huella digital ilegible ante una persona o entidad de tamaño fijo e independiente de la dimensión de los datos originales (Fuster et al., 2013).

ii) Limpieza de las variables antes de asignarlas a las consultas que se envían a la base de datos para evitar ataques por la técnica inyección SQL, en donde se realiza la inserción de una sentencia a través de los datos que se envían desde el cliente hacia la aplicación con el fin de revelar los datos del sistema, para leerlos, destruirlos o volverlos inasequibles y afectar la confidencialidad, autenticación e integridad de la información.

\section{Sistemas Distribuidos}

Los sistemas distribuidos, entendidos como una colección de computadores autónomos conectados a través de una red, que trabajan entre sí por medio del paso de mensajes para proporcionar diferentes servicios a múltiples usuarios al mismo tiempo, mediante un protocolo de comunicación mantenido por un esquema cliente-servidor, proporciona a los sistemas de información características como: escalabilidad, tolerancia a fallos y transparencia (Tanenbaum, 2009). El sistema distribuido utilizado para el desarrollo del Software, utiliza un estilo de arquitectura basado en los estándares HTTP y URL que definen un conjunto de principios para el diseño de arquitecturas que permitan una mayor escalabilidad y generalidad de interfaces, en donde cualquier cliente puede interactuar con el servidor HTTP sin ninguna configuración especial, ya que los mensajes enviados contienen toda la información necesaria para ejecutar los servicios sin que las partes necesiten recordar el estado de las comunicaciones (Sandoval, 2009).

\section{Utilización de la Aplicación}

El sistema se implantó en la empresa PetroCasinos S. A. la cual ofrece, entre otras actividades, el servicio de alimentación a empresas del sector Petrolero (Petrocasinos, 2012) y en su afán por optimizar sus servicios y disminuir sus costos, cuenta con su centro de acopio para los productos agrícolas y vio en esta herramienta la posibilidad de disminuir los eslabones de la cadena entre la producción de materia prima y la transformación de sus productos. Ellos facilitaron y permitieron la validación de la herramienta y generaron recomendaciones tales como la gestión de los precios de la oferta.

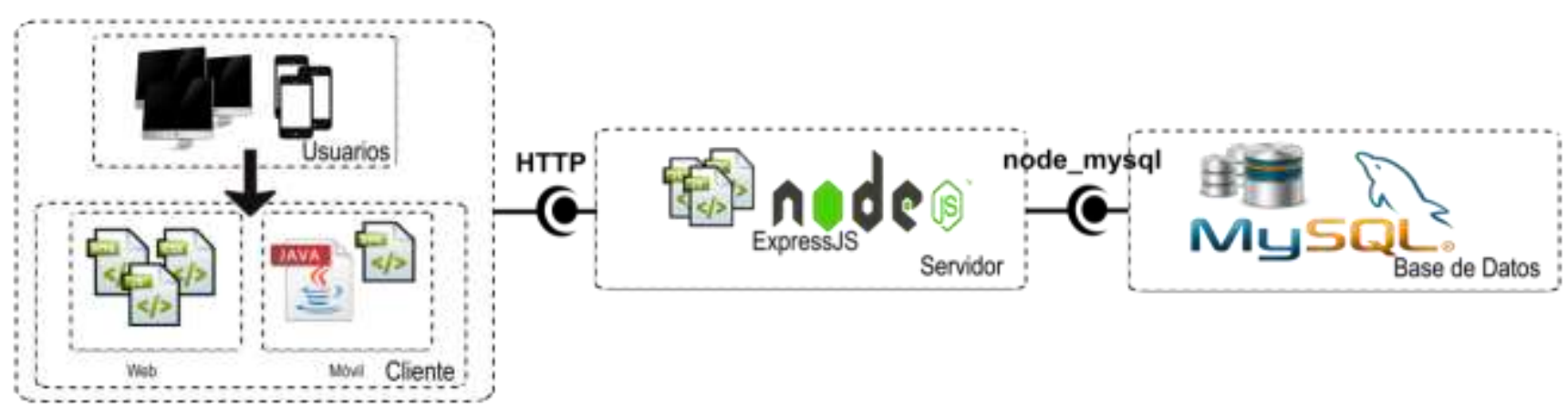

Fig. 4. Arquitectura de AGROCRAFT.

\section{CONCLUSIONES}

AGROCRAFT es una propuesta tecnológica que busca equilibrar la oferta y demanda de productos agrícolas mediante la concentración de grandes consumidores y productores agrícolas, El software permite conocer quiénes van a proveer los productos y quien los va a requerir cuando la cosecha llegue; Petrocasinos S.A optimizó sus procesos de abastecimiento, ya que a partir del uso del software concentra 
algunas productoras a quienes les compra su cosecha y sus clientes conocen con tiempo cuales son las necesidades que superaran con los cultivos, la empresa manifestó algunas recomendaciones para futuras versiones tales como el manejo de precios de comercialización, que se espera desarrollar en una futura versión.

El Diseño e implementación de la arquitectura en base a los sistemas distribuidos, facilitó el análisis de las herramientas y tecnologías necesarias para el funcionamiento del sistema y minimizó el tiempo y costo de desarrollo del software para proporcionar un sistema escalable del que se esperan futuras versiones.

El sistema de control de versiones, Subversion, permitió una mejor organización del proyecto facilitando el trabajo colectivo en lugares y tiempos diferentes, además, el desarrollo por pruebas proporcionó ventajas como especificaciones detalladas para el desarrollo y evidencia concreta del funcionamiento del software.

El modelo es viable en ambientes "ideales", por tanto se recomienda tener en cuenta variables externas que afectan la producción que permitan la definición de escenarios, la inclusión de algunas condiciones ambientales tales como condiciones ambientales, evaluaciones del terreno y actividades de administración.

\section{AGRADECIMIENTOS}

Los autores agradecen a la Universidad Pontificia Bolivariana en Bucaramanga, Colombia, por brindar las condiciones necesarias para la ejecución de las labores académicas y de investigación que permitieron esta publicación y al Ingeniero Fabio Augusto Barrera Prada de PetroCasinos S.A por verificar las funcionalidades y cumplimiento de los objetivos del Software.

\section{REFERENCIAS}

Agronet. Aplicativos móviles para el Agro Colombiano; Acceso 25 de Julio (2015). http://www.agronet.gov.co/www/htm3b/popup2uniNuke_2011.asp?cod=705 (2013)

Alaimo M; Proyectos Ágiles con Scrum: Flexibilidad, Aprendizaje, Innovación y Colaboración en Contextos Complejos. Kleer, Segunda edición, Buenos Aires (2013)

Earth Innovation Institute, Mato Grosso: Brazil's Agricultural Giant on the Pathway to State-Wide Sustainability. Acceso 30 de Enero (2016), http://earthinnovation.org/publications/mato-grosso-tps-2015/ (2015)

Erden H, Camasircioglu E, Satellite imagery acquisition planning in Agro-Geoinformatics (Agrogeoinformatics), Fourth International Conference in Agro-Geoinformaticse, 113-116 (2015).

Fuster A, Hernández L, Montoya F; Criptografía, protección de datos y aplicaciones: una guía para estudiantes y profesionales. Alfaomega, (2013)

Grande C, Orozco B; Producción y procesamiento del maíz en Colombia; Revista Científica Guillermo de Ockham Vol. 11(1), 97-110. (2013)

Lee M, Hwang J, Yoe H; Agricultural Production System Based on Internet of Things, 16th International Conference on Computational Science and Engineering (CSE), 833-837 (2013).

Llanos C, Ortiz A, Boza A; Desarrollo de una Herramienta Software para la Vista de Información de la Arquitectura CIMOSA. Inf. tecnol, 19(3), 97-106 (2008)

Mubin O, Tubb J, Novoa M, Naseem M, Razaq; Understanding the Needs of Pakistani Farmers and the Prospects of an ICT Intervention. Proceedings of the 33rd Annual ACM Conference Extended Abstracts on Human Factors in Computing Systems, 1109-1114 (2015)

OECD, Agricultural Policy Monitoring and Evaluation Acceso 30 de Enero (2016) http://dx.doi.org/10.1787/agr_pol-2012-en (2012)

PetroCasinos S. A.; PetroCasinos S. A. Acceso 25 de Julio (2015) http://www.petrocasinos.com (2015)

Sandoval J; RESTful Java Web Services. Packt Publishing, (2009)

Subversion; Control de versiones con Subversion; Acceso 28 de Febrero (2015). http://svnbook.redbean.com/nightly/es/index.html (2004)

Tanenbaum M; Sistemas Distribuidos Principios y Paradigmas. Pearson (2009) 